JOURNAL DE PHYSIQUE IV

Colloque C6, supplément au Journal de Physique III, Vol. 1, décembre 1991

C6-29

\title{
RECENT STATE OF THE THEORY OF THE METHODS OF INDUCED CONCENTRATION
}

\author{
A.V. SAMSONOVICH, V.V. SIROTKIN, N.G. USHAKOV and S.I. ZAITSEV \\ Institute of Microelectronics Technology, Academy of Sciences of USSR, Chernogolovka, \\ Moscow District 142432, USSR
}

\begin{abstract}
A set of diagnostic methods called as methods of induced concentration (MIC) results in the same signal equation. The equation allows one to solve the direct and inverse problems. The method of $Q$-variation is described and its accuracy for many-electrode EBIC is numerically estimated. Nontraditional application of MIC is argued for relief investigation.
\end{abstract}

\section{INTRODUCTION}

A set of methods in electron beam testing can be considered as one class [I] because in spite of different physical nature of measured signals they are sensitive to the concentration of charge carriers induced by the beam. The class of methods includes:

1. EBIC [2], TREBIC [3], IRBIC [4], (current)

2. EBIV [5], (potential)

3. CathodoLuminiscence $[6,7]$, (1ight intensity)

4. Microwave Absorption [8]

5. ThermoAccoustics $[9,10]$, (mechanical stress or temperature)

6. SDLTS [11], (capacity).

Usually the observed signals are related to the recombination of the induced carriers due to defects and other nonhomogeneities what allows to characterize a spatial distribution of the recombination activity after processing of measured data. If $I^{0}$ and $I(\rho,\{\phi\})$ are the signals for uniform specimen and for nonuniform one respectively then the measured contrast is given by

$$
c(\rho,\{\phi\})=\left[I^{0}-I(\rho,\{\phi\})\right] / I^{0}
$$

where $\rho=(x, y)$ are coordinates of the beam inlet, $\{\phi\}$ characterizes the inhomogeneity of the specimen (e.g. spatial distribution of the life time).

\section{SIGNAL EQUATION}

To solve direct or inverse problems it is necessary to derive so called "signal equation" which relates measured contrast with physical properties of a specimen. We consider below the case when the only one characteristic of a specimen, namely life time of the carries $\tau(r)$, is spatially dependent. Denote the nonuniform part of the life time by $\Gamma$ $\Gamma=1 / \tau-1 / \tau$

and derive the signal equation for EBIC method.

The concentration of charge carriers $P$ induced by electron or light beam is described by diffusion equation 


$$
\left\{\left[-\nabla \mathrm{D}\left(\nabla+\frac{\mu}{\mathrm{eD}}(\nabla \phi)+\frac{1}{\tau_{0}}\right]+\Gamma\right\} \mathrm{P}=\{L+\Gamma\} \mathrm{P}=\mathrm{g}\right.
$$

I denotes the content of square brackets and describes uniform situation for $\Gamma=0, g$ is the generation function. The boundary conditions are for semiinfinit space with Schottky-barrier in plane $z=0$

$$
\mathrm{s} \partial \mathrm{P} / \partial \mathrm{z}=\mathrm{P}
$$

$s$ is recombination rate. The induced current is proportional to a flow of gradient of concentration through plane $z=0$

Rewrite (1) in an integral form:

$$
I=-\int d x d z \quad D \quad \partial / \partial z
$$

$$
P(r)=P^{0}(r)+\int d V^{\prime} G^{0}\left(r, r^{\prime}\right) \Gamma\left(r^{\prime}\right) P\left(r^{\prime}\right)
$$

where $G^{0}$ and $P^{0}$ are the Green function and the solution for uniform case

$$
I G^{0}=\delta \quad(a) ; \quad L P^{0}=g \quad \text { (b) }
$$

respectively with the same boundary conditions (2). After introducing probability $Q^{0}$

$$
Q^{0}\left(r^{\prime}\right)=\int d x d y \quad D \partial G^{0}\left(r, r^{\prime}\right) /\left.\partial z\right|_{z=0}
$$

substitution of (5) into (3) gives immediately

$$
c(\rho, E)=\int \mathrm{dV} Q^{0}(r) \Gamma(r) P(r, \rho, E) / I^{0}
$$

For uniform media with diffusion length $L^{0}$ a well known result is:

$$
Q^{0}(r)=Q^{0}(z)=\exp \left(-|z| / L^{0}\right)
$$

In the case of EBIV the measured signal is a voltage on schottkycontact with nonlinear dependence on induced current [5]. However if the dependence is known and not ambiguous we come to (6) again.

For TA-contrast $[9,10]$ the signal is a mechanical stress. According to [10] the signal equation has the form of (6) in Iinear approximation and factor $Q$ can be represented as

$$
Q^{0} \propto \int d V^{\prime} d V^{\prime \prime} A_{i j}\left(r^{\prime \prime}\right)\left[\alpha_{t}\left(r^{\prime \prime}\right) \delta_{i j} \delta\left(r^{\prime \prime}-r^{\prime}\right)+s_{i j k l}\left(r^{\prime \prime}\right) \sigma_{k l}^{*}\left(r^{\prime \prime}, r^{\prime}\right)\right] T^{*}\left(r^{\prime}, r^{\prime \prime}\right)
$$

$A_{i j}$ is determined by geometry of a specimen and piezosensor, $\alpha_{t}$ is the coefficient of a temperature expansion, $s_{i j k l}$ is the tensor of elastic constants, $\sigma_{\mathrm{kl}}^{*}$ and $\mathrm{T}^{*}$ are Green functions of equations of thermoelasticity and thermodiffusion respectively [10].

In case of the microwave absorption the signal is the variance of resonator quality factor and is proportional to the number of induced carriers [8]. The factor $Q$ can be supposed to be equal to unity if $D$, $\Phi, \tau^{\circ}$ are constant along the specimen and the recombination rate $s$ is infinite.

An analyzes of a signal equation derived in [7] for cathodoluminiscence gives

$$
Q^{0}(r, \lambda)=A(z, \lambda) \sum_{i} f^{i}(\lambda) \Gamma^{i}(r) / \sum_{i} \Gamma^{i}(r)
$$

for the factor $Q^{0}$ (which is wavelength $\lambda$ dependent now). $A(z, \lambda)$ is determined by an absorption and refraction coefficients of a specimen, $f^{1}(\lambda)$ is a quantum yield of recombination of the $i-t h$ center, $1 / \Gamma^{1}(r)$ is a lifetime with respect to recombination on the $i-t h$ center [7].

As to SDLTS - contraşt, a variance of Schottky-barrier capacity measured at times $t^{1}$ and $t^{2}$ after excitation can be considered as the signal [1]]. Suppose for simplicity that $t^{1}=t^{2}=t$. Then the factor $Q$ may be represented in form (7) with substitution $t$ instead of $\lambda$ and

$$
\begin{gathered}
f^{i}(t) \simeq t^{g} \exp \left(-t / \tau^{i}\right)\left[1-\exp \left(-t / \tau^{i}\right)\right] \\
A(z)=\left(c 9 N^{0}\right)(z / h), \quad z<h
\end{gathered}
$$


$\tau^{i}$ is emission time of the $i-t h$ center, $C^{\circ}$ is nondisturbed capacity, $N^{\circ}$ is donor concentration, $h$ is its size, $t^{g}$ is excitation time.

So the signal equation can be represented in the universal form (6) in many practical cases. The concentration $P(r,(\Gamma))$ is determined by diffusion equation (1) or integral equation (3). Nonuniform part of life time $\Gamma$ provides the spatial dependence of the signal. And only the factor $Q$ is related with the method of measurement. Equation ( 6 ) is valid under some conditions e. g. condition of weak excitation what means that the induced concentration $P$ does not influence on diffusion (D), drift $(\mu ; \Phi)$, recombination $(\tau)$ and etc. More accurate analysis of the problem is given in [1]. The physical meaning of the signal equation is yield additivity of each recombination event. All these allow to joint mentioned diagnostic methods in the one class of methods of induced concentration (MIC).

\section{DIRECT PROBLEM}

The solving of a direct problem means to calculate the value of contrast using known spatial dependence of life time (or $\Gamma$ ). The inverse problem is the restoration of spatial distribution of $\Gamma$ from measured signal. A common way to solve the problems is the use of the first apprgximation [14] when the real distribution $P$ in (6) is substituted by $\mathrm{P}^{\mathrm{f}}$ from $(4 \mathrm{~b})$. An exact method of calculation of the EBIC contrast of arbitrary inclined dislocation was suggested in [12]. In [1] this method was generalized for other methods of induced concentration in the following way. Introduce in (6) by definition a function $\Gamma$

$$
c(\rho, E)=\int \mathrm{dV} Q^{0}(r) \Gamma^{\text {eff }}(r) P^{0}(r, \rho, E) / I^{0}=\int d V Q^{0}(r) \Gamma(r) P(r, \rho, E) / I^{0}
$$

Now multiply the both part of (3) on $Q^{0}(r) \Gamma^{\text {eff }}(r)$ and integrate over the space:

$$
\begin{aligned}
\int \operatorname{dVQ}^{0}(r) \Gamma^{\text {eff }}(r) & P(r)=\int \operatorname{dvQ}^{0}(r) \Gamma^{\text {eff }}(r) P^{0}(r)+ \\
& +\int d V Q^{0}(r) \Gamma^{e f f}(r) \int \operatorname{dV}^{\prime} G^{0}\left(r, r^{\prime}\right) \Gamma\left(r^{\prime}\right) P\left(r^{\prime}\right)
\end{aligned}
$$

After changing the order and variables $\left(r \Leftrightarrow r^{\prime}\right)$ of integration in the last integral of (11) and using definition (10) we obtain

$\int \operatorname{dvQ}^{0}(r) \Gamma^{\text {eff }}(r) P(r)=\int \operatorname{dvQ}^{0}(r) \Gamma(r) P(r)+$

$$
+\int d v \Gamma(r) P(r) \int d v^{\prime} Q^{0}\left(r^{\prime}\right) \Gamma^{e f f}\left(r^{\prime}\right) G^{0}\left(r^{\prime}, r\right)
$$

Equation (12) is valid for an arbitrary function $P$ therefore

$$
Q^{0}(r) \Gamma^{\text {eff }}(r)=Q^{0}(r) \Gamma(r)+\Gamma(r) \int d v^{\prime} Q^{0}\left(r^{\prime}\right) \Gamma^{\text {eff }}\left(r^{\prime}\right) G^{0}\left(r^{\prime}, r\right)
$$

so, for direct problem equation (13) determines $\Gamma^{\text {eff }}$ through $\Gamma$ and contrast can be found using (10). Equation (13) gives a regular way to construct different approximations of $\Gamma^{\mathrm{eff}}$.

A reciprocal theorem was suggested in [13] to calculate the EBICsignal and in many cases it is more convenient than the use of (10), (12). Namely this method is used below in many-electrode EBIC calculations but it is not valid for other methods of induced concentration.

\section{INVERSE PROBLEMS}

As to the inverse problem, the function $\Gamma^{\text {eff }}$ has to be found from

$$
c(\rho, E)=\int \mathrm{dV} Q^{0}(r) \Gamma^{\mathrm{eff}}(r) \mathrm{P}^{0}(r, \rho, E) / \mathrm{I}^{0}
$$

using measured signal $c$. And then $\Gamma$ can be found by simple integration, according to (13)

$$
\Gamma(r)=\frac{Q^{0}(r) \Gamma^{e f f}(r)}{Q^{0}(r)+\int d V^{\prime} Q^{0}\left(r^{\prime}\right) \Gamma^{e f f}\left(r^{\prime}\right) G^{0}\left(r^{\prime}, r\right)}
$$

For a weak inhomogeneity the integral part in denominator can be neg- 
lected in compare with $Q^{0}$ and $\Gamma=\Gamma^{\text {eff }}(r)$ as the first approximation. This result is consistent with result of Donolato [14] for EBIC. It is important to outline that any method of the numerical restoration $8 f \Gamma$, using as the first approximation equation (6) with substitution $P$ instead of $P$, gives nothing else than $\Gamma^{\text {eff }}$ and can be immediately improved with usage of (15). A couple of equations (14) and (15) gives the solution of the inverse problem not only for EBIC but also for other methods of induced concentration including CL and SDLTS in the case of one dominating type of centres. However in case of two apd more types the method of introducing of the intermediate function $\Gamma$ leads to more complex system of equations [1] for $\Gamma^{1}, f^{1}(t)$ (with substitution $\lambda$ instead of $t$ in the case of $C L$ ). According to (14) the value $F(t, r)=Q(t, r) \Gamma^{\text {eff }}(r)$ may be determined from

$$
c(t, p, E)=\int d V F(t, r) \quad P^{0}(r, \rho, E) / T^{0}
$$

A system of equation for $\Gamma^{i}, f^{i}(t)$ is

$$
\begin{gathered}
\sum_{i}\left[A(2, t) f^{i}(t)-B(r, t)\right] \Gamma^{i}(r)=F(t, r) \\
B(r, t)=\int d V^{\prime} F\left(r^{\prime}, t\right) G^{0}\left(r^{\prime}, r\right)
\end{gathered}
$$

And finally consider the expansion of solution method on the time resolved methods (TREBIC and so on) [1]. Define the signal as

$$
c(p, t)=\left[I^{0}(t)-I(p, t)\right] /\left\langle I^{0}\right\rangle ; \quad\left\langle I^{0}\right\rangle=\int_{0}^{T} d t \quad I^{0}(t) / T
$$

It is determined by a nonstationar diffusion equation

$$
\left\{\frac{\partial}{\partial t}+\left[-\nabla D\left(\nabla+\frac{\mu}{\mathrm{eD}}(\nabla \phi)+\frac{1}{\tau^{0}}\right]+\Gamma\right\} \mathrm{P}=\left\{\frac{\partial}{\partial t}+\mathrm{L}+\Gamma\right\} \mathrm{P}=\mathrm{g}\right.
$$

which comes after Laplace-transformation

to a stationary form

$$
f(t) \Rightarrow f(s)=\int d t \exp (-s t) f(t)
$$

$$
\{\partial / \partial t+L+\Gamma\} \Rightarrow\{s+L+\Gamma\}
$$

and transformation parameter $s$ plays a role of the inverse life time and may be used as an additional variable instead of beam energy $E$ in stationery case (14), (15). For time resolved methods we obtain the following form of the inverse problem [1]

$$
\begin{gathered}
c(\rho, s)=\frac{1}{\left\langle I^{0}>\right.} \int d v Q^{0}(r, s) \Gamma^{\text {eff }}(r, s) P^{0}(r, \rho, s) \\
\Gamma(r)=\frac{Q^{0}(r, s) \Gamma^{e f f}(r, s)}{Q^{0}(r, s)+\int d V^{\prime} Q^{0}\left(r^{\prime}, s\right) \Gamma^{e f f}\left(r^{\prime}, s\right) G^{0}\left(r^{\prime}, r, s\right)}
\end{gathered}
$$

However now these equations can not be solved consequently because $\Gamma^{\text {eff }}$ depends on four variables and can not be found from (18). Ways of solving (18), (19) are discussed in [1].

\section{METHOD OF Q-VARIATION (MANY-ELECTRODE EBIC)}

Return to eq (11)-(13). The basic idea of the reconstruction was to measure signal varying position $(\rho)$ and (using $E$ ) size of induced carrier cloud. Another way is to vary probability $Q$. For EBIC the method of Q-variation was suggested in [12] as many-electrode EBIC with application to the measurement of the generation function. The simplest arrangement consists of two schottky-contacts with narrow slot between them. Neglecting for a moment with diffusion in compare to drift, the depletion region is divided into two collection volumes so that each electrode collects carriers from correspondent volume only. A diffusion "washes out" this ideal picture resulting in loss of accuracy. Quantitative results are shown on Fig. 1. The curve OB represents the isoline on which conditional probability to reach the left electrode is equal 
to a half, OA is a isoline of 0.8 -probability, OC represents the level of 0.2-probability. If a collection volume contains a small inhomogeneity then the collected current will be influenced by the size and the position of the inhomggeneity. Fig.1 shows that using different bias voltages $U^{\text {eff }}$ and $U^{r i g t h}$ it is possible to manage with "neutral" line settling the inhomogeneity in different collection volumes so that first inhomogeneity influences on collected current of one electrode and then on another one. Varying simultaneously $\mathrm{U}^{\text {left }}$ and $\mathrm{U}^{\mathrm{rigth}}$ it is possible to estimate the depth of the inhomogeneity whereas a changing of one of voltages allows to estimate an azimuth. A distance between $O A$ and $O C$ represents a lateral resolution which has submicron value. Dashed lines $O D, O E$ and $O F$ are 0.5 levels and correspond to different values of voltage demonstrating that the whole depletion region may be swept.

\section{NONTRADITIONAL APPLICATIONS (RELIEF AND ROUGHNESS INVESTIGATION)}

Consider a uniform specimen restricted by surface relief $z=f(\rho)$. Let each induced couple gives contribution into a signal with some constant probability $\mathrm{W}$. Then the signal is

$$
I(\rho, E)=\underset{z<f}{W} \int_{f V} g(r, \rho, E)
$$

Thus the signal is proportional to absorbed in specimen energy of the electron beam. For small reljef the generation function can be written as $g\left(x, \rho^{0}\right)=g\left(x-x^{0}, y-y^{0}, z-f\left(x^{0}, y^{0}\right), E\right)$ where $\rho=\left(x^{0}, y^{0}\right)$ and now after substitution $z^{\prime}=z-f\left(x, y^{\prime}\right)$ we obtain

$$
I\left(P_{0}, E\right)=w \int_{-\infty}^{\infty} d x \int_{\infty}^{\infty} d y \int_{-\infty}^{0} d z^{\prime} g\left(x-x^{0}, y-y^{0}, z^{\prime}+f(x, y)-f\left(x^{0}, y^{0}\right), E\right)
$$

An expansion for small $f$ gives

$$
I\left(\rho^{0}, E\right)=W \int_{-\infty}^{\infty} d x \int_{\infty}^{\infty} d y \int_{-\infty}^{0} d z \cdot g\left(x-x^{0}, y-y^{0}, z^{\prime}, E\right)+
$$

$+W \int_{-\infty}^{\infty} d x \int_{\infty}^{\infty} d y \int_{-\infty}^{0} d z, \frac{\partial}{\partial z} g\left(x-x^{0}, y-y^{0}, z^{\prime}, E\right)\left[f(x, y)-f\left(x^{0}, y^{0}\right)\right]+$

$+w \frac{1}{2} \int_{-\infty}^{\infty} d x \int_{\infty}^{\infty} d y \int_{-\infty}^{0} d z^{\prime} \frac{\partial^{2}}{\partial z^{2}} g\left(x-x^{0}, y-y^{0}, z^{\prime}, E\right) \quad\left[f(x, y)-f\left(x^{0}, y^{0}\right)\right]^{2}+\ldots$

Assume for simplicity that $\langle f\rangle=\int_{-\infty}^{\infty} d x \int_{\infty}^{\infty} d y f(x, y)=0$. Then the mean value of the signal is:

$$
<I>=\int_{-\infty}^{\infty} d x^{0} \int_{\infty}^{\infty} d y^{0} I\left(\rho^{0}, E\right)=\left\langle I^{0}>+W \int_{\infty}^{\infty} d x \int_{\infty}^{\infty} d y \int_{-\infty}^{0} d z g_{z z}(x, y, z, E)[K(x, y)-K(0,0)]\right.
$$

This expression allows to calculate mean value of signal using such statistical characteristic of random relief as correlation function $\mathrm{K}(\mathrm{x}, \mathrm{y})$ and what is more important to reconstruct the correlation function measuring the mean value of difference $\left\langle I-I^{0}\right\rangle$ as function of beam energy E. Such situation is a consequence of specifig dependence of generation function on the relief shape $f$, namely $g\left(\rho^{0}, z-f\left(\rho^{0}\right)\right)$. The dependence is not valid for example for light excitation of bulk specimen but can occur for films. CL, TA and absorption of VHF power are ideal methods for such measurement whereas EBIC is not so convenient due to dependence of $W$ on generation point of an electron-hole couple with respect to collecting electrodes or junctions.

Another possibility arises if the signal equation (20) is used for restoration of nonrandom relief itself. As for backscattered and secondary electron signals [15] a simple model of signal formation (gene- 
ration function $g$ is taken as Gaussian) describes very well the $a b-$ sorbed energy signal in compare to Monte-Carlo simulation. Fig. 2 . shows also the result of relief reconstruction according to the methods and algorithms developed in [15] for BSE-signal.

Fig. 1

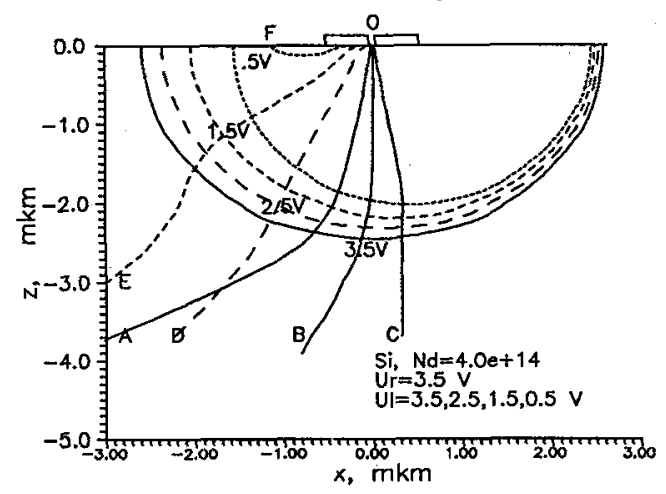

Fig.2

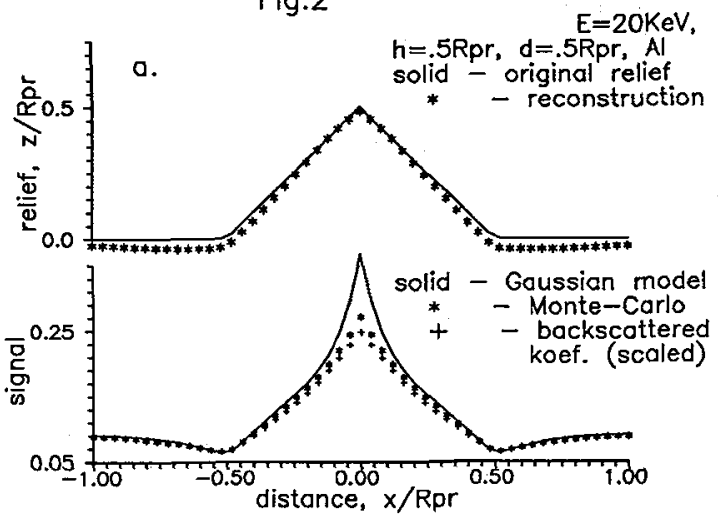

Fig.1 The calculation of charge collection probability in manyelectrode EBIC. "Neutral" lines $O B, O D, O E$, $O F$ divide space into two collection volumes and correspond to different values of bias voltage of the left electrode.

Fig.2 The coefficient of backscattered energy is calculated using MC-model $((*)$ on Fig.2b)) and Gaussian model (solid line on Fig.2b)) for the relief shown on Fig.2a by solid line, the heigth $h$ and base $2 d$ are measured in unit of practical range $\mathrm{R}^{\mathrm{pr}}$. The result of the restoration is shown on Fig. 2 a by $(*)$.

\section{REFERENCES}

1. Zaitsev S I, Samsonovich A V, Izvestia AN SSSR, ser. fiz., 1990, v.54, P. 247-254.

2. Leamy H J, J. Appl. Phys., 1982, V 53, P. R51.

3. George A, Fournier J M, Bois D, Scan.Elec.Micr.,1982, V 19, p 147.

4. Castaldini A, Cavaldini A, Gondi P, Izvestia AN SSSR, ser. fiz., 1987 , v.51, P. 1547.

5. Rau E I, Sasov A V, spivak G W, Dziesiaty J, Wencel K, Phys. Stat. Sol. (a), 1982, v 71, p. 429.

6. Spivak G V, Petrov V I, Antoshin M K, Usp.Fiz.Nauk, 1986, V 148, p689

7. Hegert $W$, Paseman $L$, Phys. Stat. Sol. (a), 1984, V 85, p. 641 .

8. Milaev V A, Sanina V A, Izv. vuz., ser. radiofiz., 1980, v 23, p 407.

9. Rozencwaig A, Gersho A, J. Appl. Phys., 1976, V 47, p 64.

10. Jackson W, Amer N M, J. Appl. Phys., 1980, v 51, p 3343 .

11. Breitenstein 0 , scanning, 1985, v 7, p 273.

12. Zaitsev S I, Samsonovich A V, Izvestia AN SSSR, ser. fiz., 1987, $\mathrm{v} 51, \mathrm{p} 1547$.

13. Donolato C, J. Appl. Phys., v 66, pp 4524-4525.

14. Donolato $c$, optic, 1978, v 52, p 19.

15. Firsova A A, Reimer I, Ushakov N G, Zaitsev S I, Scanning, 1991 (in press). 